\title{
Using NMR to study full intact wine bottles
}

\author{
A.J. Weekley, P. Bruins, M. Sisto, and M.P. Augustine* \\ Department of Chemistry, University of California, One Shields Avenue, Davis, CA 95616, USA
}

Received 6 August 2002; revised 19 November 2002

\begin{abstract}
A nuclear magnetic resonance (NMR) probe and spectrometer capable of investigating full intact wine bottles is described and used to study a series of Cabernet Sauvignons with high resolution ${ }^{1} \mathrm{H}$ NMR spectroscopy. Selected examples of full bottle ${ }^{13} \mathrm{C}$ NMR spectra are also provided. The application of this full bottle NMR method to the measurement of acetic acid content, the detection of complex sugars, phenols, and trace elements in wine is discussed.
\end{abstract}

(C) 2003 Elsevier Science (USA). All rights reserved.

Keywords: Wine; Bottle; Ethanol; Acetic acid; Trace element

\section{Introduction}

The unique ability of nuclear magnetic resonance (NMR) spectroscopy to non-destructively and non-invasively provide one-to-one relationships between measured spectra and chemical structure has moved NMR to the forefront of chemical structure determination techniques [1]. It is well known that a simple recipe involving a host of multi-dimensional pulse sequences, will yield the three-dimensional molecular connectivity for a sample containing just one chemical compound [2]. Unfortunately, this methodology breaks down when applied to complex mixtures of chemical compounds as the structure for each of the separate molecules in the mixture is not automatically obtained. The protocol typically adopted in this case first involves separating the mixture into each molecular component. The structure for each of these separate compounds is then determined with NMR spectroscopy. This approach of course fails if the mixture is contained in a sealed vessel or can not be separated into its molecular constituents. Even though the measurement of molecular level structural details is not possible in this more restrictive application, NMR spectroscopy has had some success relating spectral intensities to material properties [3-5].

\footnotetext{
${ }^{*}$ Corresponding author. Fax: 1-530-752-8995.

E-mail address: augustin@chem.ucdavis.edu (M.P. Augustine).
}

Given this success, it is natural to extend NMR towards characterizing a valuable commodity like wine. A necessary first step in this study is the identification of wine industry issues that can potentially be addressed with NMR spectroscopy. Some of these problems include determining the effects of wine on health [6,7], wine origin [8], the type, source, and extent of wine spoilage [9-11], the presence of cork taint in wine [12], the concentration and type of sulfides in wine [13], the concentration, source, and type of trace metal contaminants in wine [14], the concentration and type of phenols in wine [15], and finally the relationship between dilute molecular components like aldehydes and flavenoids and wine properties such as age, bouquet, and texture [15]. NMR spectroscopy has already been used to study a limited subset of these problems in detail and most studies either exploit the natural abundance ${ }^{2} \mathrm{H}$ NMR of native wine samples [16-19] or apply standard multidimensional NMR pulse sequences to molecules isolated from wine [20,21]. Natural abundance ${ }^{2} \mathrm{H}$ NMR has been used to both track the origin of and validate wines. This fingerprinting method compares experimentally determined intensity ratios from the ${ }^{2} \mathrm{H}$ NMR spectrum for the HOD, $-\mathrm{CHD}-$, and $-\mathrm{CH}_{2} \mathrm{D}$ peaks for the water and ethanol in wine to a database of intensity ratios for known wines $[16,17]$. It is not immediately obvious that these peak ratios will change for different wines, however since the natural abundance of the ${ }^{2} \mathrm{H}$ isotope in rainfall intimately depends upon location on the surface 
of the Earth and water is used during grape growth, the amount of deuterium in water is propagated from the grape, to the must, and finally during fermentation to the ethanol. Clearly any augmentation of the wine during fermentation by for example chaptalization or the addition of sugar to increase alcohol content will change how the deuterium in water is incorporated into ethanol. In fact, chaptalization is illegal in the warmer climate wine producing regions of California, South Africa, and Chile [18] and has been studied in detail with natural abundance ${ }^{2} \mathrm{H}$ NMR by the Joint Research Center in Italy [19]. The NMR of wine has not just been limited to the ${ }^{2} \mathrm{H}$ nucleus, rather multi-dimensional NMR spectroscopy has also been used to identify the trace amino acids in wine and to determine the structure of trace organic molecules in wine [20,21]. This class of experiments has also been applied to the study of wine aging. Here the tannins that are responsible for the taste and longevity of red wine were examined closely in order to characterize the slow chemical reactions that modify the tannin chemical structure [22].

The recurring theme that ties all of the NMR studies to date together is the sample size. Typically high-resolution liquid state NMR samples with volume on the order of $1 \mathrm{~mL}$ or less are used in commercially available narrow bore NMR spectrometers. The obvious requirement of this approach is that either the wine bottle seal must be broken in order to extract a sample or that the wine must be obtained prior to bottle corking. One goal of this paper is to describe an NMR spectrometer and probes that remove this requirement and instead investigate full intact wine bottles. Operation in this way does not violate the bottle seal, labeling, or corking thus the contents of extremely valuable wine bottles can be fingerprinted and authenticated without having to open the bottle and thus devalue the wine.

An application of the full bottle method introduced here to wine spoilage is discussed following a description of the NMR spectrometer and probes necessary to accomplish full bottle studies of wine. It is well known that wine spoils due to the generation of vinegar produced by the oxidation of ethanol to acetic acid catalyzed by acetic acid bacteria or acetobacter in the presence of oxygen [23]. Many of these and other bacteria survive all of the steps of wine making from the mature grape through vinification to bottle corking [24]. The presence of oxygen initiates the production of acetic acid and there are many different ways that oxygen can be introduced into the wine bottle. Improper corking procedures as well as leaky, faulty, or old corks lend themselves to oxygen leakage. All of these effects are more likely to influence the quality of more valuable aged wines. The standard method of acetic acid quantitation in wine first involves separation of the acetic acid from wine via steam distillation. The concentration of acetic acid in the distillate is subsequently determined by a titration with a standardized sodium hydroxide solution [15]. Of course the more modern analytical tools including gas chromatography, liquid chromatography, and ultraviolet/visible spectroscopy are also often used to characterize the amount of acetic acid in bottled wine [15,25], although the predominate approach involves distillation. Again, much like in all previous NMR experiments involving wine, all of these acetic acid quantitation techniques require that the wine bottle be violated to extract samples. The non-destructive and non-invasive NMR determination of acetic acid content in wine is straightforward given that an NMR probe capable of investigating full bottle samples is available and that the static magnetic field over the entire bottle is homogeneous enough to resolve the $\approx 1 \mathrm{ppm}$ chemical shift difference between the acetic acid methyl group protons and the ethanol methyl group protons [26]. Here water, ethanol, and acetic acid comprise the major proton containing components in the full bottle of spoiled wine. Therefore measurement of the intensity of these various peaks in the standard ${ }^{1} \mathrm{H}$ NMR spectrum is enough information to determine the degree of wine spoilage.

The NMR instrumentation presented here is versatile and not just limited to studies involving ${ }^{1} \mathrm{H}$ NMR spectroscopy. Rather any heteronucleus having an adequate chemical shift dispersion between the bulk wine and a target compound is a likely candidate. The flexibility of the instrumentation is demonstrated by investigating ${ }^{13} \mathrm{C}$ NMR spectra for full bottles of wine and red wine vinegar. Finally, possible extensions to other NMR active isotopes important in enology are discussed.

\section{Experimental}

Full bottle acetic acid standards were prepared by mixing variable volumes of $99.7 \%$ glacial acetic acid obtained from E.M. Science (Gibbstown, NJ) with a $12.5 \%(\mathrm{v} / \mathrm{v})$ solution in water of 200 proof ethyl alcohol purchased from Gold Shield Chemical Company (Hayward, CA). Sodium chloride obtained from Fisher Scientific was dissolved into $750 \mathrm{~mL}$ of water and was used as a calibration standard for both shimming the magnetic field for nuclei with low gyromagnetic ratio $\gamma$ and for determining the Larmor frequency of the comparatively less sensitive ${ }^{13} \mathrm{C}$ nucleus in full bottle wine samples. All of the wines tested in this study were obtained from the UC Davis wine library located in the Department of Viticulture and Enology.

All NMR experiments were performed at $2.01 \mathrm{~T}$ using an Oxford Instruments $310 \mathrm{~mm}$ room temperature bore superconducting solenoid imaging magnet. Careful adjustment of both the cryogenic and room temperature magnetic field shims establishes an extremely homogeneous magnetic field over the entire wine bottle leading 
to $4 \mathrm{~Hz}$ wide ${ }^{1} \mathrm{H}$ lines. Current for the room temperature shims was provided by a General Electric Omega series NMR spectrometer magnetic field shim power supply modified to output -5 to $+5 \mathrm{~V}$ DC on each channel. The supply was controlled by a potentiometer bank salvaged from a Varian EM $39090 \mathrm{MHz}$ continuous wave NMR spectrometer. Careful adjustment of these room temperature shims compensate for differences between all common wine bottle shapes, sizes, and corks. Lead or metallic bottle seals do not measurably interfere with the probe tuning or the homogeneity and intensity of the rf field across the wine bottle.

\section{1. ${ }^{1} H$ NMR spectroscopy}

The location of the wine bottle and NMR probe with respect to the superconducting solenoid imaging magnet used for ${ }^{1} \mathrm{H}$ NMR spectroscopy is shown in Fig. 1(a).
An exploded view of the NMR probe used for recording ${ }^{1} \mathrm{H}$ spectra for full intact wine bottles at a Larmor frequency of $85.78 \mathrm{MHz}$ that shows the location of the wine bottle with respect to the rf detection coil is also included in Fig. 1(a). Here the rf coil examines the neck of the wine bottle between the base of the cork and the main body of the wine bottle. Although there is less sample in this region of the bottle in comparison to the bottle body and base, it is much easier to both tune and match a small volume rf coil to the high ${ }^{1} \mathrm{H}$ Larmor frequency as well as to establish a homogeneous static magnetic field over the small sample region to ultimately produce narrow highly resolved NMR lines.

Even though it is an extremely complex mixture of many diverse chemical compounds, wine displays a rather simple ${ }^{1} \mathrm{H}$ NMR spectrum. In the absence of spoilage, the ${ }^{1} \mathrm{H}$ NMR spectrum obtained following single pulse excitation using the sequence provided in
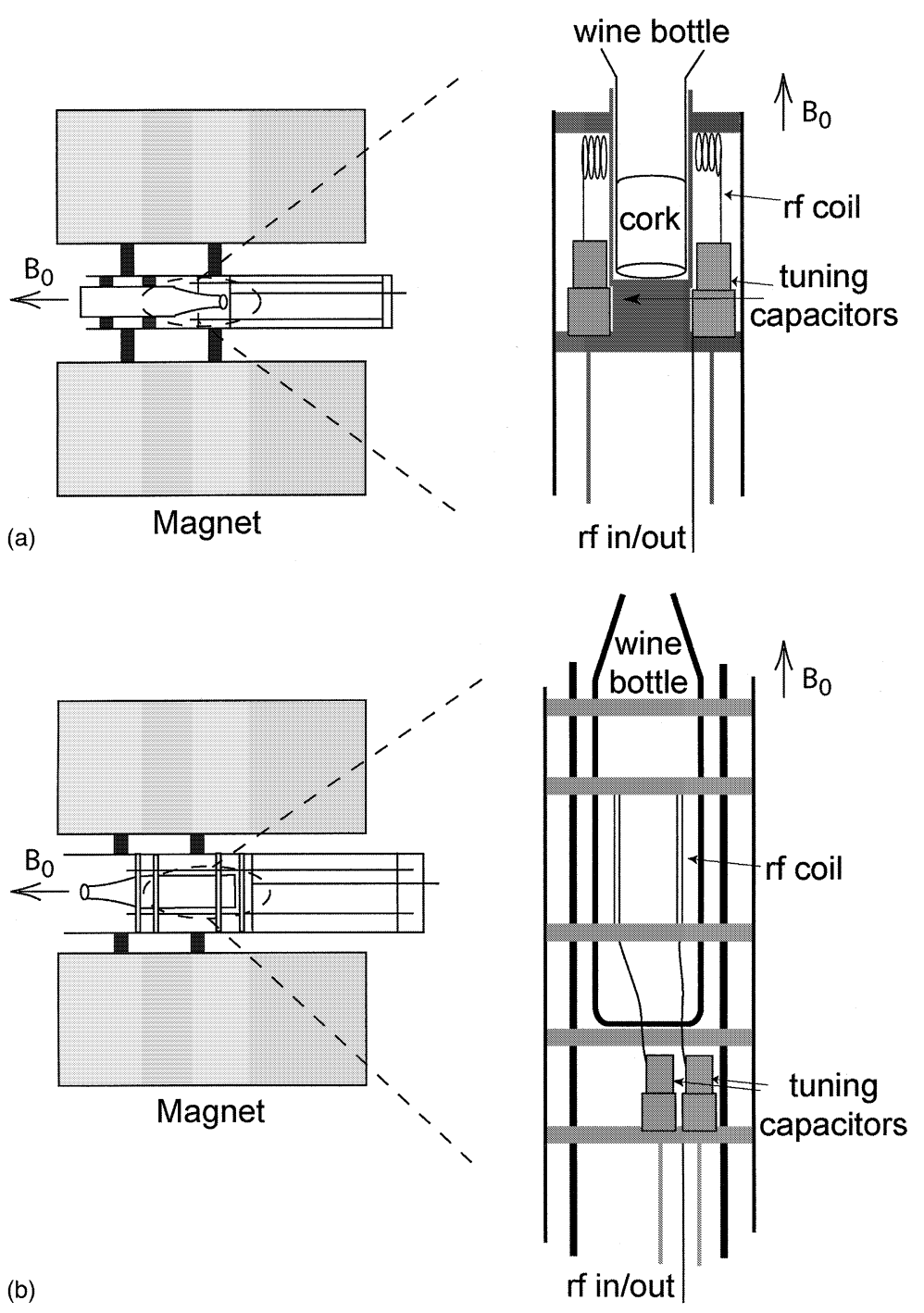

Fig. 1. Experimental setup used to obtain the NMR spectrum of full intact wine bottles in a horizontal bore magnetic resonance imaging magnet. The exploded views shown in (a) and (b) show the NMR probe heads used to measure ${ }^{1} \mathrm{H}$ and ${ }^{13} \mathrm{C}$ NMR spectra in full wine bottles, respectively. To ensure high resolution NMR spectra, the bottles are slightly tilted to prevent air bubbles from settling in the rf coil region. 
(a)

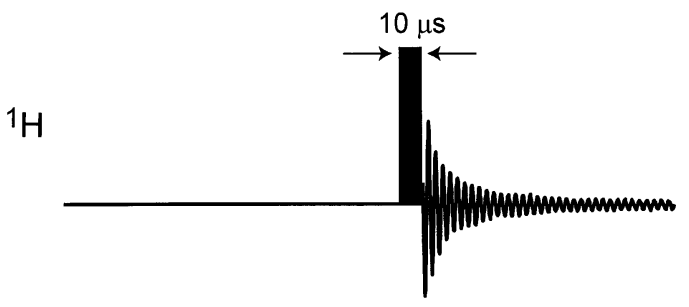

(b)

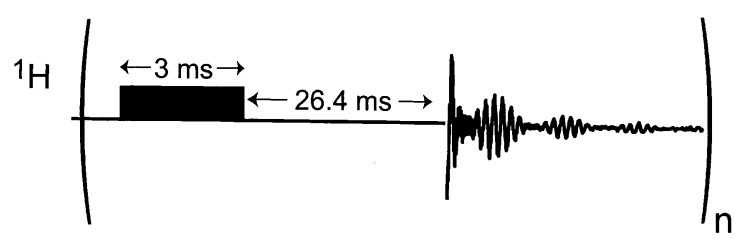

(c)

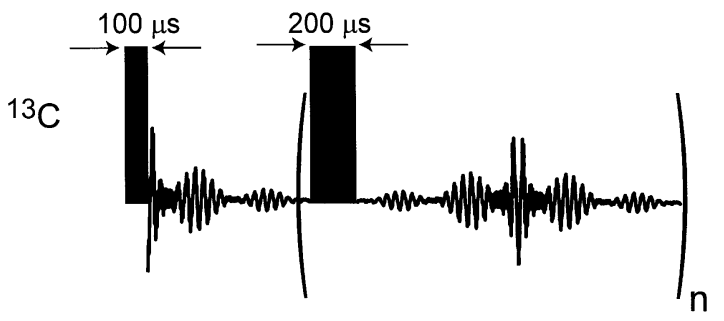

Fig. 2. Pulse sequences used to measure full bottle NMR spectra and quantify acetic acid contamination in wine.

Fig. 2(a) has a singlet centered at $4.8 \mathrm{ppm}$ corresponding to water and a quartet and triplet centered at 3.6 and $1.1 \mathrm{ppm}$, respectively, due to ethanol. The generation of low levels of acetic acid upon spoilage yields another singlet in the ${ }^{1} \mathrm{H}$ NMR spectrum centered at $2.1 \mathrm{ppm}$. Taking the ratio of the integrated intensities of the ethanol triplet to the water peak and the acetic acid peak to the ethanol triplet as $f_{\mathrm{EtOH}}$ and $f_{\mathrm{HOAc}}$, respectively, allows the percentage of ethanol by volume and the concentration of acetic acid in wine to be quantified as

$\mathrm{EtOH} \%(\mathrm{v} / \mathrm{v})=\frac{f_{\mathrm{EtOH}} \times 10^{3}}{\left(8.5+8.2 f_{\mathrm{HOAc}}\right) f_{\mathrm{EtOH}}+4.6}$

and

$[\mathrm{HOAc}](\mathrm{g} / \mathrm{L})=\frac{f_{\mathrm{HOAc}} f_{\mathrm{EtOH}} \times 10^{4}}{\left(8.3+8.0 f_{\mathrm{HOAc}}\right) f_{\mathrm{EtOH}}+4.5}$,

where the molecular weights and densities of water, ethanol, and acetic acid have been used to produce the numbers in the denominators. The measurement of $f_{\mathrm{EtOH}}$ is simple from the one pulse experiment shown in Fig. 1(a), however, an estimate of $f_{\mathrm{HOAc}}$ using standard one pulse excitation is complicated by the strong water and ethanol signals comprising roughly $99 \%$ of the spectral intensity. Recognizing that the methyl group resonances for ethanol and acetic acid are centered at 1.1 and $2.1 \mathrm{ppm}$, respectively, and that water is shifted $2.7 \mathrm{ppm}$ or $232 \mathrm{~Hz}$ downfield from the acetic acid peak at $2.01 \mathrm{~T}$, prompts application of the pulse sequence shown in Fig. 2(a). Here a combination of selective ex- citation, delayed acquisition, and block averaging are used to reliably and reproducibly measure $f_{\mathrm{HOAc}}$. The $3 \mathrm{~ms}$ long soft $\mathrm{rf}$ pulse tips the water magnetization by less than $5^{\circ}$ and when combined with a $200 \mathrm{~Hz}$ audio filter bandwidth attenuates the water peak by three orders of magnitude. The delayed acquisition combined with the long spin-spin relaxation times for the ethanol and acetic acid methyl group protons removes the short life time free induction decay components that lead to broad lines thus yielding extremely narrow $4 \mathrm{~Hz}$ wide peaks. Since the magnetic field is not stabilized with a flux locked loop and an internal ${ }^{2} \mathrm{H}$ lock is not possible for sealed wine bottles, block averages of $n=10$ groups of 100 scans are typically measured. The $n=10$ separate free induction signals are Fourier transformed, overlapped by shifting the frequency, and added off line using Matlab. In this way long time drift in the static magnetic field is eliminated thereby producing highly resolved ${ }^{1} \mathrm{H}$ NMR spectra for the methyl group region in wine that can be used to accurately measure $f_{\mathrm{HOAc}}$.

\section{2. ${ }^{13} \mathrm{C}$ and low $\gamma$ nuclei}

A schematic showing the location of the wine bottle in the low frequency NMR probe and the placement of the probe with respect to the superconducting solenoid imaging magnet is provided in Fig. 1(b). An additional exploded view of this probe shows the location of the $\mathrm{rf}$ excitation and detection coils with respect to the full wine bottle. The much wider chemical shift range and lower Larmor frequency of $21.56 \mathrm{MHz}$ at $2.01 \mathrm{~T}$ for ${ }^{13} \mathrm{C}$ in comparison to ${ }^{1} \mathrm{H}$ relaxes the requirement for an extremely narrow line width and hence removes the volume constraint recognized in the design presented in Fig. 1(a). Consequently the rf detection coil is centered on the main body of the wine bottle to improve sensitivity without sacrificing rf coil filling factor.

The formation of spin echoes for low $\gamma$ nuclei is possible using the probe shown in Fig. 1(b), although the geometry of the four turn split solenoid rf coil is not optimized for homogeneity. In the special case of ${ }^{13} \mathrm{C}$ NMR spectroscopy where spin-lattice and spin-spin relaxation times are long, the multiple $\pi$ pulse sequence provided in Fig. 2(c) is used to refocus magnetization and increase the signal-to-noise ratio for a fixed number of scans by offline adding the free induction signal following the $100 \mu \mathrm{s} \pi / 2$ pulse to the echo signals appearing at $102 \mathrm{~ms}$ intervals. In this way fully ${ }^{1} \mathrm{H}$ coupled ${ }^{13} \mathrm{C}$ NMR spectra for full bottle samples corresponding to $100-1000$ scans can be obtained in a reasonable period of time.

\section{Results and discussion}

Examples of the performance of the probe shown in Fig. 1(a) and the pulse sequence provided in Fig. 2(b) 


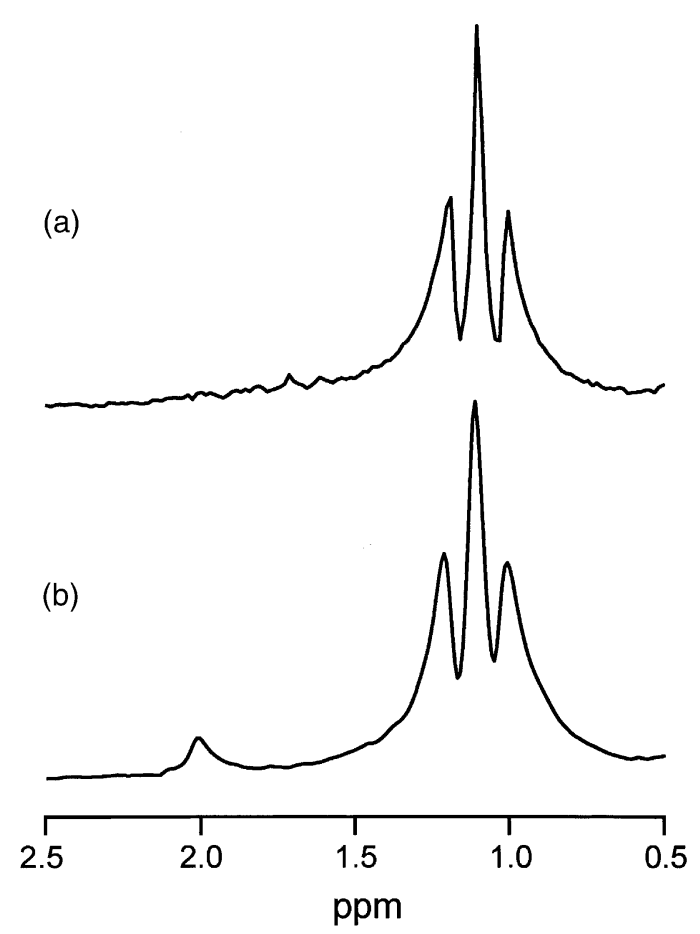

Fig. 3. ${ }^{1} \mathrm{H}$ NMR spectra obtained at $2.01 \mathrm{~T}$ for a full intact bottle of the 1997 vintage UC Davis Cabernet Sauvignon in (a). The spectrum shown in (b) corresponds to a full bottle of $12.5 \%(\mathrm{v} / \mathrm{v})$ ethanol in water with an added $5.25 \mathrm{~g} / \mathrm{L}$ of acetic acid.

are given in Fig. 3. The ${ }^{1} \mathrm{H}$ NMR spectrum corresponding to the methyl group region of a full bottle of the 1997 vintage UC Davis Cabernet Sauvignon is shown in Fig. 3(a) while the ${ }^{1} \mathrm{H}$ spectrum for a full bottle having $12.5 \%(\mathrm{v} / \mathrm{v})$ ethanol dissolved into water with $0.5 \%(\mathrm{v} / \mathrm{v})$ added acetic acid is shown in Fig. 3(b). It is clear that the triplet in these spectra corresponds to the methyl group in ethanol judging from both the $1.1 \mathrm{ppm}$ chemical shift and the splitting pattern due to scalar coupling with the two equivalent methylene ${ }^{1} \mathrm{H}$ nuclei in the ethanol chemical structure. The single peak at $2.1 \mathrm{ppm}$ in the spectrum shown in Fig. 3(b) corresponds to acetic acid. Eq. (1) can be rearranged in the usual limit that the prepared concentration of ethanol in water is $12.5 \%(\mathrm{v} / \mathrm{v})$ and that $f_{\mathrm{HOAc}}$ is much smaller than one to yield $f_{\mathrm{EtOH}}=6.4 \times 10^{-2}$. The ratio of the integrated intensity of the acetic acid peak to the ethanol triplet shown in Fig. 3(b) gives $f_{\mathrm{HOAc}}=4.5 \times 10^{-2}$ which can be used in Eq. (2) to calculate the concentration of acetic acid as $[\mathrm{HOAc}]=5.7 \mathrm{~g} / \mathrm{L}$, a number that compares well with the prepared $5.3 \mathrm{~g} / \mathrm{L}$ of acetic acid in the $0.5 \%(\mathrm{v} / \mathrm{v})$ standard preparation. The $7 \%(0.4 \mathrm{~g} / \mathrm{L})$ deviation between these concentration measurements is most likely due to an error in the standard preparation.

In most practical situations there is no prior knowledge of the ratio $f_{\mathrm{EtOH}}$ because many wines of different vintages, sources, types, and quality can have an ethanol concentration between 7 and $24 \%$ (v/v) [15]. Here the pulse sequence presented in Fig. 2(a) is first used to measure the entire ${ }^{1} \mathrm{H}$ NMR spectrum followed by application of the sequence shown in Fig. 2(b) to selectively excite and detect the methyl group region. In this way both $f_{\mathrm{EtOH}}$ and $f_{\mathrm{HOAc}}$ can be measured from peak integrals and in combination with Eqs. (1) and (2) yield the percentage of ethanol by volume and the concentration of acetic acid. The accuracy and sensitivity of this approach to acetic acid quantitation has been tested in full bottles by comparing NMR derived concentrations to the actual prepared concentration [27]. The one-to-one agreement between the different concentration measurements with the less than $0.1 \mathrm{~g} / \mathrm{L}$ acetic acid sensitivity of the full bottle NMR approach prompts the study shown in Fig. 4. Here the NMR derived percent ethanol and acetic acid concentration in a vertical series of sealed full bottles of the UC Davis Cabernet Sauvignon in the time period between 1950 and 1977 are compared. As expected, the amount of ethanol in this Cabernet Sauvignon does not correlate well with year and varies between ten and twenty percent. It is interesting to note that the two most recent vintages display concentrations of ethanol very close to the current $12.5 \%(\mathrm{v} / \mathrm{v})$ industry average for most wines. A similar lack of correlation with age is observed in Fig. 4(b) for the full bottle acetic acid concentrations for these same wines. Although the oldest wine displays the largest $6.3 \mathrm{~g} / \mathrm{L}$ acetic acid spoilage and the youngest wine
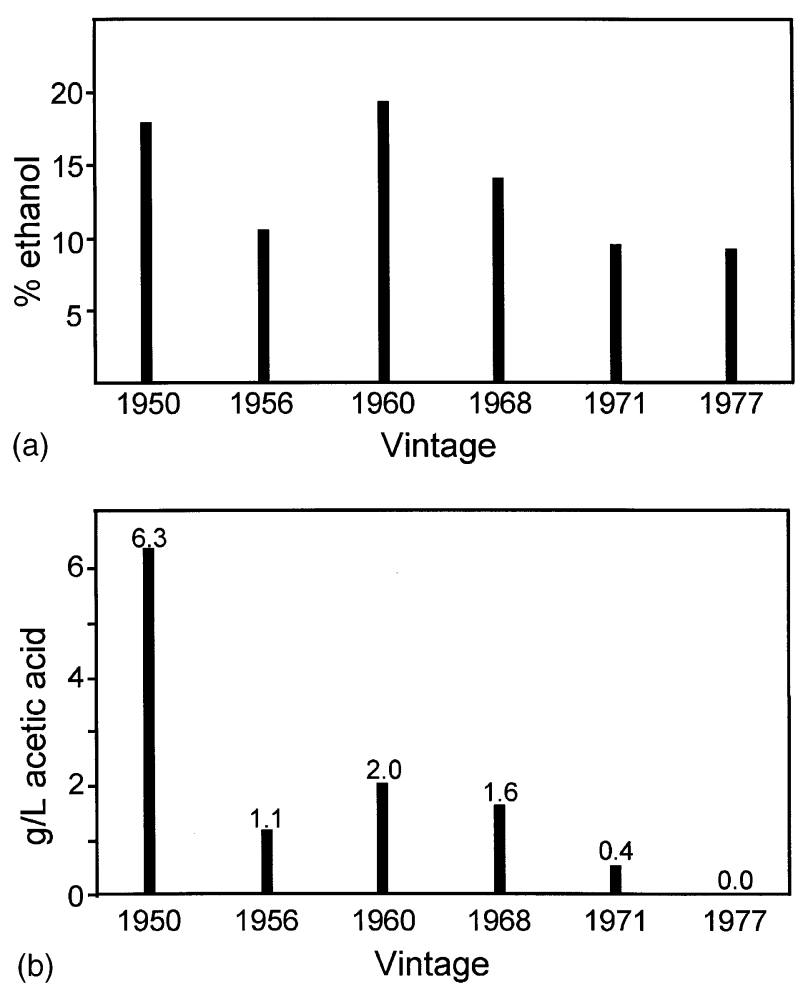

Fig. 4. Histograms summarizing the ethanol content in (a) and acetic acid content in (b) measured for full bottles of the UC Davis Cabernet Sauvignon dating back to 1950 . 
apparently has no measurable acetic acid contamination, the acetic acid concentration in the other vintages between 1956 and 1971 varies between 0.4 and $2.0 \mathrm{~g} / \mathrm{L}$. It is therefore incorrect to assume that older wines will automatically have a higher acetic acid concentration than younger wines. The integrity of the cork and hence the quality of the bottle seal against oxygen leakage with time is of paramount importance to acetic acid contamination.

It is impossible to quantify cork sealing performance without violating the contents of the bottle in the absence of this full bottle NMR approach. In many cases opening the wine bottle is not an option because the seal becomes damaged and the wine is seriously devalued. The danger and accuracy of visual inspection of cork sealing performance are illustrated in Figs. 5(a and b) for the 1950 and 1977 vintage UC Davis Cabernet Sauvignon, respectively. Both of the cork seals in these pictures appear to be seriously compromised, however, comparison to the results provided in Fig. 4(b) reveals that only the 1950 vintage displays severe acetic acid spoilage. This result suggests that at some point the 1977 vintage wine leaked its contents but, only a small

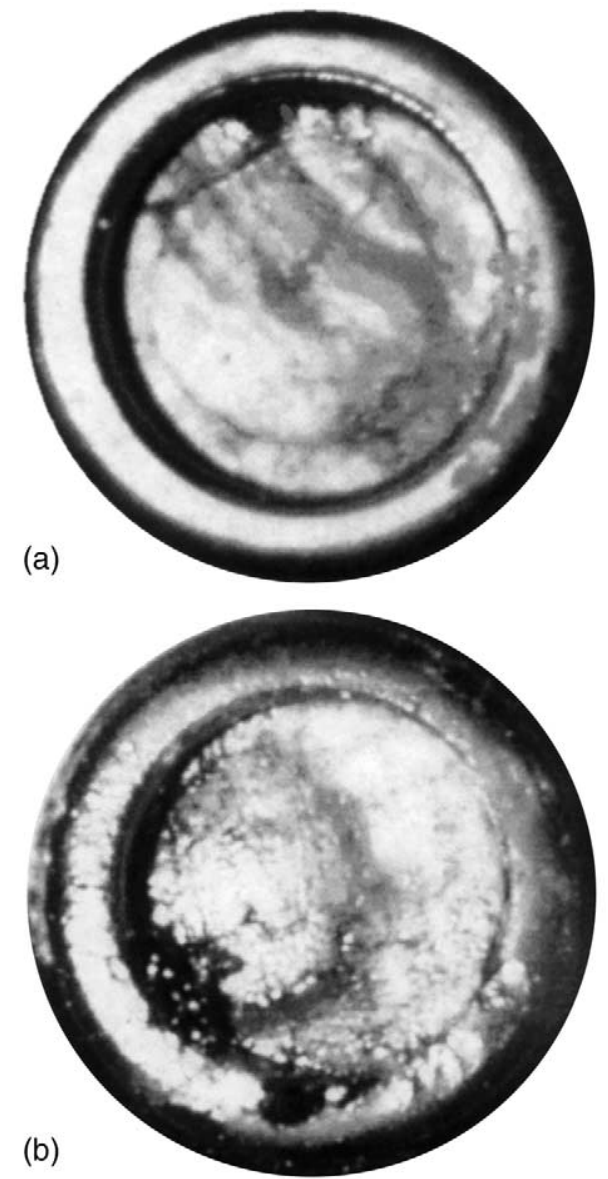

Fig. 5. Pictures of the corks used to seal the 1950 (a) and 1977 (b) vintages of the UC Davis Cabernet Sauvignon. amount of oxygen leaked into the bottle. The liquid leak in the cork most likely sealed before the large quantity of oxygen necessary to oxidize ethanol into measurable quantities of acetic acid was admitted to the bottle.

A potential problem with the experimental setup shown in Fig. 1(a) is the limitation to examine only the bottle neck. As mentioned above the tradeoff between sensitivity, resolution, and circuit tuning motivated the rf coil placement around the neck. Fortunately there are no detrimental effects due to the presence of the lead or metallic seals around the bottle neck. Any slight changes in the rf tank circuit tuning or ${ }^{1} \mathrm{H}$ NMR line width due to the metallic conductor is easily compensated by adjusting the tuning and matching capacitors or the room temperature shims.

In an effort to increase the sensitivity of the measurement of other dilute components like flavenoids and aldehydes in wine as well as extend the full bottle technique to other nuclei with lower gyromagnetic ratio and thus lower Larmor frequencies, the probe shown in Fig. 1(b) was constructed. Instead of examining the $\approx 25 \mathrm{~cm}^{3}$ sample volume in the neck of the wine bottle as shown in Fig. 1(a), the probe design presented in Fig. 1(b) investigates the $\approx 1 \mathrm{~L}$ volume in the body of the wine bottle. The factor of forty increase in measured sample more than compensates for the factor of eight loss in sensitivity upon reducing the Larmor frequency by a factor of four. Although the magnetic field homogeneity is much worse across the larger sample volume, examination of nuclei like ${ }^{13} \mathrm{C}$ with a larger chemical shift dispersion than ${ }^{1} \mathrm{H}$ will be less sensitive to the increased line width. The increased sample volume also has the added benefit of making ${ }^{13} \mathrm{C}$ NMR on sealed full wine bottles feasible for the first time. The ${ }^{13} \mathrm{C} N M R$ spectrum provided in Fig. 6(a) produced from the pulse sequence shown in Fig. 2(c) with $n=7$ corresponds to the full bottle of the 1997 vintage UC Davis Cabernet Sauvignon. The triplet and quartet centered at 57 and $18 \mathrm{ppm}$, respectively, are due to the ethanol methylene and methyl ${ }^{13} \mathrm{C}$ nuclei. The line splitting of $\approx 140 \mathrm{~Hz}$ in both of these peaks and their respective splitting pattern is consistent with scalar coupling to directly bonded ${ }^{1} \mathrm{H}$ nuclei. Another demonstration of the viability of full bottle ${ }^{13} \mathrm{C}$ NMR studies of wine and its molecular constituents is shown in Fig. 6(b). Here a full bottle of red wine vinegar is used to examine the additional ${ }^{13} \mathrm{C}$ NMR peaks due to the carbonyl and methyl groups in acetic acid centered at 178 and $21 \mathrm{ppm}$, respectively. The inverted triangles provided in Fig. 6(b) label the acetic acid methyl group quartet. The near equal integrated intensity of these nested quartets suggests that the amount of ethanol and acetic acid in this red wine vinegar sample are nearly equal, a result consistent with the literature [28].

It is clear from the spectra shown in Fig. 6 that the full bottle ${ }^{13} \mathrm{C}$ NMR method is feasible but, perhaps, not 

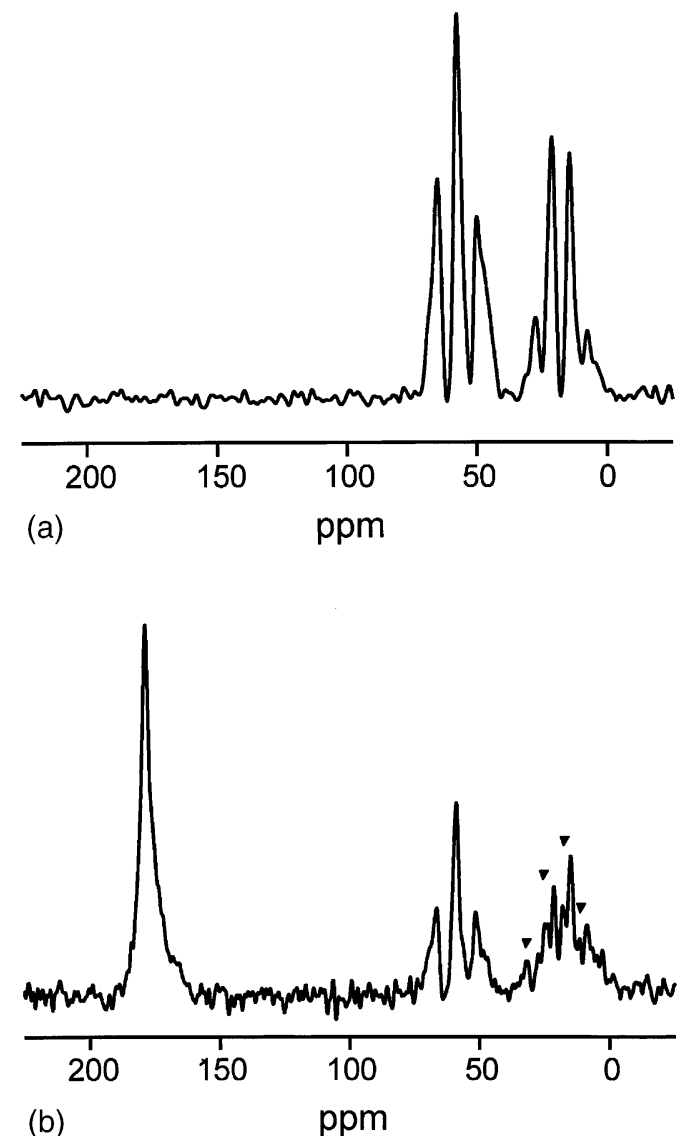

Fig. 6. Full bottle ${ }^{13} \mathrm{C}$ NMR spectrum for the 1997 vintage UC Davis Cabernet Sauvignon shown in (a) obtained with the NMR probe presented in Fig. $1 \mathrm{~b}$ and the pulse sequence provided in Fig. 2c with $n=7$. The ${ }^{13} \mathrm{C}$ NMR spectrum shown in (b) corresponds to a similar full bottle sample of home made red wine vinegar. The inverted triangles provided in (b) label the methyl group quartet for acetic acid.

practical for the more interesting exploration of vital wine components like tannins, flavenoids, phenols, and aldehydes. In principle continued signal averaging will eventually reveal these peaks in ${ }^{13} \mathrm{C}$ NMR spectra, but, without decoupling the spectra will be very complicated and difficult to analyze. In addition, the nuclear Overhauser enhancement recognized during ${ }^{1} \mathrm{H}$ decoupling is not provided in the current single resonance probe designs. A future goal is to incorporate an additional ${ }^{1} \mathrm{H}$ channel to the low frequency full bottle probe shown in Fig. 1(b) in order to provide the increased resolution and sensitivity realized by ${ }^{1} \mathrm{H}$ decoupling. This effort will make full bottle multi-dimensional ${ }^{13} \mathrm{C}$ NMR spectroscopy of dilute wine components a real practical objective.

Finally it is important to note that the NMR of wine is not limited to just $I=1 / 2$ nuclear spins like ${ }^{1} \mathrm{H}$ and ${ }^{13} \mathrm{C}$. Rather one could imagine quantifying the amount of trace elements in full bottle samples. Given that NMR can reliably fingerprint small wine samples as mentioned above, an obvious direction involves full bottle ${ }^{2} \mathrm{H}$ NMR wine validation. Here the need for violating the sample contents is removed while the NMR authentication is unaffected. Other potential candidates for NMR spectroscopy in wine include isotopes like ${ }^{207} \mathrm{~Pb},{ }^{199} \mathrm{Hg},{ }^{45} \mathrm{Sc},{ }^{39} \mathrm{~K},{ }^{27} \mathrm{Al}$, and ${ }^{23} \mathrm{Na}$, etc. Although the abundance of these isotopes in wine is typically below the detection limit for standard $500 \mu \mathrm{L}$ volume NMR samples, the increased volume recognized by the probe shown in Fig. 1(b) amplifies the number of spins by a factor of $10^{4}$ thus making the study of trace elements in native wine samples accessible to NMR spectroscopy for the first time. Furthermore, full bottle studies are not just limited to the wine itself. The quality and nature of the wine bottle could be explored by a combination of ${ }^{29} \mathrm{Si}$ and ${ }^{23} \mathrm{Na}$ NMR spectroscopy while the cork, either natural or synthetic, could be studied with ${ }^{13} \mathrm{C}$ solid-state NMR techniques.

\section{Conclusion}

The purpose of this paper was to extend NMR spectroscopy to the analysis of enological problems in full bottle wine samples. It is important to realize that the full bottle NMR approach is applicable to any type of wine even though the only examples presented here include the Cabernet Sauvignons. The specific problem of acetic acid spoilage in wine addressed in this study provides one of perhaps more NMR based handles on cork sealing performance. It is hoped that the full bottle NMR method will become routine in the evaluation and authentication of the quality of valuable fine wines. The extension of the full bottle method to the study of more dilute wine components, trace ionic contaminants, and other wine related problems where specific chemical products are produced or used promises to be an exciting future direction.

\section{Acknowledgments}

The gift of the UC Davis Cabernet Sauvignons from the UC Davis Cellar Master Ernie Farinias and help with the cover art by Gary Johnson and Diana HirningJohnson is gratefully acknowledged. M.P.A. is a David and Lucile Packard and an Alfred P. Sloan Foundation Fellow.

\section{References}

[1] R.R. Ernst, Principles of Nuclear Magnetic Resonance in One and Two Dimensions, Oxford University Press, New York, 1987.

[2] K. Wüthrich, NMR Of Proteins and Nucleic Acids, Wiley, New York, 1986.

[3] K.Z. Qin, D.Y. Chem, Z.G. Li, A new method to estimate the oil and gas potentials of coals and kerogens by solid state C-13 NMR spectroscopy, Org. Geochem. 17 (1991) 865-872. 
[4] S. Zaidi, Coal reactivity-correlations with fuel ratio and NMR data, Fuel Process Technol. 41 (1995) 253-259.

[5] A. Adhvaryu, J.M. Perez, L. Duda, Quantitative NMR spectroscopy for the prediction of base oil properties, Tribol. Trans. 43 (2000) 245-250.

[6] A.A. de Lorimier, Alcohol, wine, and health, Am. J. Surg. 180 (2000) 357-361.

[7] J.B. German, R.L. Walzem, The health benefits of wine, Annu. Rev. Nutr. 20 (2000) 469-474.

[8] J. AiresDeSousa, Verifying wine origin: a neural network approach, Am. J. Enol. Viticult. 47 (1996) 410-414.

[9] M. de Toit, I.S. Pretorius, Microbial spoilage and preservation of wine: using weapons from nature's own arsenal: a review, S. Afr. J. Enol. Viticult. 21 (2000) 74-96.

[10] D. Sohier, A. Lonvaud-Funel, Rapid and sensitive in situ hybridization method for detecting and identifying lactic acid bacteria in wine, Food Microbiol. 15 (1998) 391-397.

[11] P. Kalathenos, J.P. Sutherland, T.A. Roberts, Resistance of some wine spoliage yeasts to combinations of ethanol and acids present in wine, J. Appl. Bacteriol. 78 (1995) 245-250.

[12] C. Silva Pereira, J.J. Figueiredo Marques, M.V. San Romao, Cork taint in wine: scientific knowledge and public perception: a critical review, Crit. Rev. Microbiol. 26 (2000) 147-162.

[13] M. Mestres, M.P. Marti, O. Busto, J. Guasch, Simultaneous analysis of thiols, sulphides and disulphides wine aroma by headspace solid-phase microextraction-gas chromatorgaphy, J. Chrom. A 849 (1999) 293-297.

[14] L. Sauvage, D. Frank, J. Stearne, M.B. Millikan, Trace metal studies of selected white wines: an alternative approach, Anal. Chim. Acta 458 (2002) 223-230.

[15] B.W. Zoecklein, K.C. Fugelsang, B.H. Gump, F.S. Nury, Wine Analysis and Production, Aspen, Gaithersburg, MD, 1995.
[16] G.J. Martin, M.L. Martin, F. Mabon, M. Michon, Identification of the origin of natural alcohols by natural abundance hydrogen-2 nuclear magnetic resonance, Anal. Chem. 54 (1982) 2380-2382.

[17] G.J. Martin, M.P. Day, B. Zhang, Determination of the geographical origin of wine using joint analysis of elemental and isotopic composition, J. Sci. Food Agric. 67 (1995) 113-123.

[18] J. Priewe, Wine from Grape to Glass, Abbeville Press, New York, 1999.

[19] C. Guillou, F. Reniero, Magnetic resonance sniffs out bad wine, Phys. World 11 (1998) 22-23.

[20] J. Kidric, I. Kosir, M. Kocjancic, Wine analysis by 1D and 2D NMR spectroscopy, Analysis 26 (1998) 97-101.

[21] J. Kidric, I.J. Kosir, Use of modern nuclear magnetic resonance spectroscopy in wine analysis: determination of minor compounds, Anal. Chim. Acta 458 (2002) 77-84.

[22] C. Saucier, I. Pianet, M. Laguerre, Y. Glories, NMR and molecular modeling: application to wine ageing, J. Chim. Phys. 95 (1998) 357-365.

[23] G.S. Drysdale, G.H. Fleet, Acetic acid bacteria in winemaking: a review, Am. J. Enol. Viticult. 39 (1988) 143-154.

[24] P. Ribereau-Gayon, New developments in wine microbiology, Am. J. Enol. Viticult. 36 (1985) 1-10.

[25] R. Kellner, R. Schindler, R. Vonach, B. Lendl, A rapid method for wine analysis based upon sequential injection (SI)-FTIR spectroscopy, Fresen. J. Anal. Chem. 362 (1998) 130-136.

[26] MIT web page, Department of Chemistry Instrumentation Facility. Available from http://web.mit.edu/speclab/www/nmr_chart.html.

[27] A.J. Weekley, P. Bruins, M.P. Augustine, A nondestructive method of determining acetic acid spoilage in an unopened bottle of wine, Am. J. Enol. Vitic. (in press).

[28] P. Jackisch, Modern Winemaking, Cornell University Press, Ithaca, NY, 1985. 